\title{
A Novel Image Denoising using Matched Partial Biorthogonal Wavelets and Adaptive Thresholding
}

\author{
Prashant Bhati \\ Dept. of Digital Communication \\ SSSIST SEHORE, India
}

\author{
Prof. Mukesh Tiwari \\ Dept. of Digital Communications \\ SSSIST SEHORE, India
}

\begin{abstract}
The denoising of a natural image corrupted by Gaussian noise is a problem in signal or image processing Even though much work has been done in the field of wavelet thresholding, most of it was focused on statistical modeling of wavelet coefficients and the optimal choice of thresholds. This paper describes a new method for suppression of noise in image by fusing the wavelet Denoising technique with optimized thresholding function, improving the denoised results significantly by using partial biorthogonal wavelets. In today's scenario denoising techniques use the classical orthonormal wavelets for decomposition of an image corrupted with additive white Gaussian noise, upon which various thresholding strategies are built. We present a method to design partial biorthogonal wavelet bases and report on their potential for denoising. This paper describes a new method for suppression of noise in image by fusing the wavelet Denoising technique with optimized thresholding function, improving the denoised results significantly. Simulated noise images are used to evaluate the denoising performance of proposed algorithm along with another wavelet-based denoising algorithm. Experimental result shows that the proposed denoising method outperforms standard wavelet denoising techniques in terms of the PSNR and the preservation of edge information. The use of available biorthogonal wavelets in image denoising is less common because of their poor performance. But when we combine the approach of fusion with partial biorthogonal wavelets then the performance is increases in comparison of traditional methodology. This point to the importance of matching when using wavelet-based denoising.
\end{abstract}

\section{Keywords}

Biorthogonal wavelets, Thresholding, Denoising

\section{INTRODUCTION}

The wavelet transform has been a powerful and widely used tool in image denoising because of its high energy compaction and multi resolution properties. Denoising an image corrupted with additive white Gaussian noise was initially proposed in [1] by thresholding the wavelet coefficients. Subsequently, various decomposition strategies and thresholding schemes were developed which have been proposed in [2], [3], [4]. But, the problem remain same most of these use classical orthogonal wavelets which are independent of the image and noise characteristics and focus on finding the best threshold. Unlike the Fourier transform with its complex exponential basis, the wavelet transforms do not have a unique basis. Noting this point several attempts at designing matched wavelets have been made with the goal of match varying from match to a signal [5] and energy compaction [6] to maximizing the signal energy in the scaling sub-space [7]. In this paper, we assume the noise to be additive white Gaussian and use the statistical properties of noise in the design.
Many scientific data sets are contaminated with noise either because of data acquisition process or because of naturally occurring phenomenon. One classical task of image processing is to discriminate between noise and signal, and to remove the unwanted noise from the signal. Especially for the case of additive white Gaussian noise a number of techniques using wavelet-based thresholding have been proposed. Donoho and Johnston proposed hard and soft thresholding methods for Denoising. This scheme exterminates many wavelet coefficients that might contain useful image information. However, the major problem with both methods is the choice of a suitable threshold value. The definition of coefficient independent threshold given by Donoho and Johnston depends on the noise power and the size of the image. In practice, however one deals with images of finite size where the applicability of such a theoretical result is rather questionable. In addition, most signals show a spatially non-uniform energy distribution, which motivates the choice of a non-uniform threshold. Besides wavelet-thresholding, many other approaches have been suggested as well. For example wavelet-based denoising using Hidden Markov Trees, which was initially proposed by Crouse. has been quite successful, and it gave rise to a number of other HMT-based schemes. They tried to model the dependencies among adjacent wavelet coefficients using the HMT, and used the minimum mean-squared error (MMSE)-like estimators for suppressing the noise [8][9][10]. Specifically, we propose a design that will generate a partial biorthogonal wavelet bases for a given corrupted image which can be used for its denoising.

The remaining of this paper is organized as follows. We discuss Adaptive matched Partial biorthogonal wavelets in Section 2. In Section 3 we discuss about Wavelet Thresholding . The proposed method working process in section 4 . In section 5 we discuss about the Challenges with corresponding discussion. The conclusions and future directions are given in Section 6. Finally references are given.

\section{ADAPTIVE MATCHED PARTIAL BIORTHOGONAL WAVELETS}

We use the concept of separable kernel proposed by Mallat [11] in our design of matched wavelets for images. Hence, two sets of 1D matched wavelets are designed for two 1-D signals generated from the given image, obtained by row and column orderings instead of designing the two dimensional matched wavelets. We have opted for separable kernel mainly for simplicity of the design procedure. Now the problem of finding image-matched biorthogonal wavelets is essentially one of designing 2-channel 1D FIR perfect reconstruction filter bank for each of the two 1-D signals satisfying some necessary and sufficient conditions. The design of FIR filters makes the obtained wavelets to be compactly supported. Our criterion for matching is based on maximizing the projection of signal characteristics into the scaling subspace rather than the wavelet subspace, based on 
knowledge of the noise characteristics. Such a matching criteria will lead to a higher SNR in the coarsest approximation subspace when a noisy signal is decomposed with the matched as opposed to a fixed wavelet. Thus a thresholding process which passes the coarsest approximation sub-band and attenuates the rest of the sub-bands should decrease the amount of residual noise in the overall signal after the denoising process. A similar approach was proposed by Gupta et al. [12] for designing wavelets for signal and image compression. However, their wavelets would not work for the denoising applications as they are matched directly to the input image which is a noisy image in denoising applications i.e their technique would maximize the projection of noisy image features into the scaling subspace and not the clean image features. Next, we present our design in detail.

Often in digital signal processing the need arises to decompose signals into low and high frequency bands, after which need to be combined to reconstruct the original signal. Such an example is found in subband coding (SBC). This demo shows an example of perfect reconstruction of a two-channel filter bank, also known as the Quadrature Mirror Filter (QMF) Bank since it uses power complementary filters. We will simulate our perfect reconstruction process by filtering a signal made up of Kronecker deltas. Plots of the input, output, and error signal will be provided, as well as the magnitude spectra of the signals. The mean-square error will also be computed to measure the effectiveness of the perfect reconstruction filter bank.

Perfect reconstruction is a process by which a signal is completely recovered after being separated into its low frequencies and high frequencies. Below is a block diagram of a perfect reconstruction process which uses ideal filters. The perfect reconstruction process requires four filters, two lowpass filters (H0 and $\mathrm{G} 0$ ) and two highpass filters (H1 and $\mathrm{G} 1)$. In addition, it requires a downsampler and upsampler between the two lowpass and between the two highpass filters. Note that we have to account for the fact that our output filters need to have a gain of two to compensate for the preceding up sampler.

\section{Perfect Reconstruction}

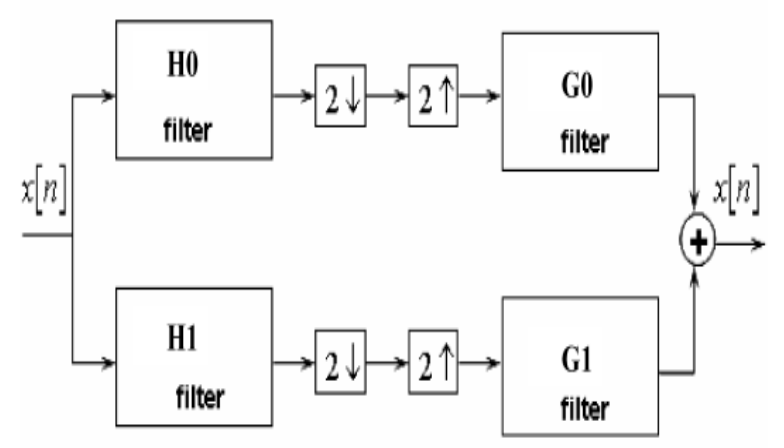

All filters are ideal

\section{Fig 1 Perfect Reconstruction}

The Filter Design Toolbox ${ }^{\mathrm{TM}}$ provides a specialized function, called FIRPR2CHFB, to design the four filters required to implement an FIR perfect reconstruction two-channel filter bank as described above. FIRPR2CHFB designs the four FIR filters for the analysis ( $\mathrm{H} 0$ and $\mathrm{H} 1$ ) and synthesis (G0 and $\mathrm{G} 1$ ) sections of a two-channel perfect reconstruction filter bank. The design corresponds to so-called orthogonal filter banks also known as power-symmetric filter banks, which are required in order to achieve the perfect reconstruction.

An adaptive image coding method and system are disclosed. The system accepts an input image, divides it into image segments, and assigns each segment to a wavelet transform filter from a bank of such filters for transformation. The bank preferably comprises filters adapted for different types of image content, e.g., sharp edges, slowly-varying contours, etc. Filters are preferably assigned so as to produce minimal distortion for their assigned image segments at a given target bit rate, each filter produces transform coefficients for its segment using scale and subband settings common to the entire image. The valid coefficients for each segment are then combined in a composite wavelet coefficient image, which resembles a single wavelet transform of an entire image-although different filters are used to create different portions of the coefficient image. The composite image allows joint, rate-distortion optimized coding for a segmented image. Joint coding allocates bits between the transforms of the image segments optimally, and produces an easily scaleable bit stream.

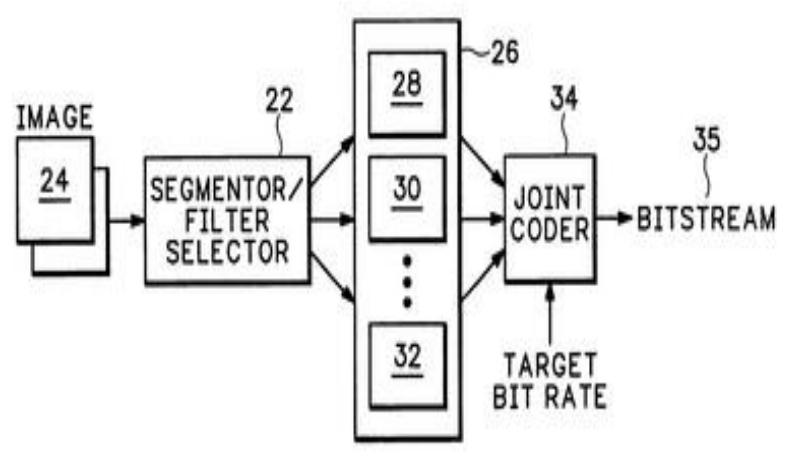

Fig 2 Adaptive Reconstruction

\section{WAVELET THRESHOLDING}

Wavelet Thresholding is a simple non-linear technique, which operates on one wavelet coefficient at a time. In its most basic form, each coefficient is threshold by comparing against threshold, if the coefficient is smaller than threshold, set to zero; otherwise it is kept or modified. Replacing the small noisy coefficients by zero and inverse wavelet transform on the result may lead to reconstruction with the essential signal characteristics and with less noise. Wavelet thresholding involves threes steps A linear forward wavelet transform, nonlinear thresholding step\& a linear inverse wavelet transform. Let us consider a signal $\{i j x, j i,=1,2 \ldots \mathrm{M}\}$ denote the $\mathrm{M} \mathrm{X} \mathrm{M}$ matrix of the original image to be recovered and $\mathrm{M}$ is some integer power of 2.During transmission the signal is corrupted by independent and identically distributed (i.i.d) zero mean, white Gaussian Noise $i j z$ with standard deviation $\sigma$ i.e $i j z j \sim N$ $(0, \sigma 2)$ as follows.

$$
y_{i j}=x_{i j}+z_{i j}
$$

From this noisy signal $i j y$, we want to find an approximation $i j$ $x$.The goal is to estimate the signal ij $x$ from noisy observations ij $y$ such that Mean Squared error (MSE) is minimum. ie

$$
\|X-\bar{X}\|^{2} \quad=\frac{1}{N} \sum_{i=0}^{N-1}\left|x_{i}-\overrightarrow{x_{i}}\right|^{2}
$$


Let $\mathrm{W}$ and $\mathrm{W}-1$ denote the two-dimensional orthogonal discrete wavelet transform (DWT) matrix and its inverse respectively. Then the above equation can be written as

$$
d_{i, j}=c_{i j}+\varepsilon_{i j}
$$

With $=d W y, c=W x, \varepsilon=W z$. Since $W$ is orthogonal transform, $j \varepsilon$ is also an i.i.d Gaussian random variable with $i j \varepsilon \approx(0$, $\sigma 2)$.Now $T($.$) be the wavelet thresholding function then the$ wavelet thresholding based Denoising scheme can be expressed as $x=W-1(T(W y))$

Wavelet transform of noisy signal should be taken first and then thresholding function is applied on it. Finally the output should be undergone inverse wavelet transformation to obtain the estimate $x$. There are four thresholds frequently used, i.e. hard threshold, soft threshold, semi-soft threshold, and semi-hard threshold. The hard-thresholding function keeps the input if it is larger than the threshold; otherwise, it is set to zero. It is described as

$$
\begin{gathered}
f_{h}(x)=x \text { if } x \geq \lambda \\
=0 \text { otherwise }
\end{gathered}
$$

The hard-thresholding function chooses all wavelet coefficients that are greater than the given threshold $\lambda \psi$ and sets the others to zero. The threshold $\lambda$ is chosen according to the signal energy and the noise variance $2 \sigma$. If a wavelet coefficient is greater than $\lambda$, we assume that as significant and attribute it to the original signal. Otherwise, we consider it to be due to the additive noise and discard the value. The soft-thresholding function has a somewhat different rule from the hardthresholding function. It shrinks the wavelet coefficients by $\lambda \psi$ towards zero, which is the reason why it is also called the wavelet shrinkage function.

$$
\begin{aligned}
f_{s}(x) & =x-\lambda & & \text { if } x \geq \lambda \\
& =0 & & \text { if } x<\lambda \\
& =x+\lambda & & \text { if } x \leq-\lambda
\end{aligned}
$$

The soft-thresholding rule is chosen over hard-thresholding, for the soft-thresholding method yields more visually pleasant images over hard thresholding.

\section{PROPOSED METHOD \\ WORKING PROCESS}

AND

Let's design a filter bank with filters of order 99 and pass band edges of the lowpass and highpass filters of 0.45 and 0.55 , respectively:

$\mathrm{N}=99$;

$[\mathrm{H} 0, \mathrm{H} 1, \mathrm{G} 0, \mathrm{G} 1]=\operatorname{firpr} 2 \mathrm{chfb}(\mathrm{N}, .45)$;

Note that the analysis path consists of a filter followed by a downsampler, which is a decimator, and the synthesis path consists of an upsampler followed by a filter, which is an interpolator. So, we can use the multirate filter objects available in the Filter Design Toolbox to implement our analysis and synthesis filter bank by using a decimator followed by an interpolator, respectively.

$\%$ Analysis filters (decimators).

$\mathrm{Hlp}=$ mfilt.firdecim $(2, \mathrm{H} 0)$;

Hhp = mfilt.firdecim $(2, \mathrm{H} 1)$;

$\%$ Synthesis filters (interpolators).

$\mathrm{Glp}=$ mfilt.firinterp $(2, \mathrm{G} 0)$;

Ghp = mfilt.firinterp $(2, \mathrm{G} 1)$;
Looking at the first lowpass filter we can see that it meets our 0.45 cutoff specifications.

hfv $=$ fvtool(Hlp);

legend(hfv,'Hlp Lowpass Decimator');

set(hfv, 'Color', [ [ $\left.\left.\begin{array}{lll}1 & 1 & 1\end{array}\right]\right)$

Finding an optimized value $(\lambda)$ for thresholding is a major problem. A small threshold will surpass all the noisy coefficients so the denoised signal is still noisy. Conversely a large threshold value makes more number of coefficients as zero which leads to smooth signal and destroys details that may cause blur and artifacts. So, optimum threshold value should be found out, which is adaptive to different subband characteristics. Here, we describe an efficient method for fixing the threshold value for denoising by analyzing the statistical parameters of the wavelet coefficients. This customized thresholding function which is similar to the soft thresholding function but with a smooth transition around $\lambda$. The thresholing function we proposed as

$$
\begin{array}{rlrl}
f_{c}(x) & =x-\operatorname{sgn}(x)(1-\alpha) \lambda & & \text { if }|x| \geq \lambda \\
& =0 & & \text { if }|x| \leq \gamma \\
& =\alpha \lambda\left(\frac{|x|-\gamma}{\lambda-\gamma}\right)^{2}(\alpha-3)\left(\frac{|x|-\gamma}{\lambda-\gamma}\right)+4-\lambda \text { otherwise }
\end{array}
$$

Where $0<\lambda \gamma<$ and $01 \leq \leq \alpha$. This idea is similar to that of the semi soft or firm shrinkage proposed by Gao and Bruce, and the non-negative garrote thresholding function suggested by Gao, in the sense that they are continuous at $\lambda \psi$ and can adapted to the signal characteristics. In the thresholding function ( ) $\mathrm{x} \mathrm{fc}, \gamma \psi$ is the cut-off value, below which the wavelet coefficients are set zero, and $\alpha \psi$ is the parameter that decides the shape of the thresholding function ( ) $\mathrm{x} f$.

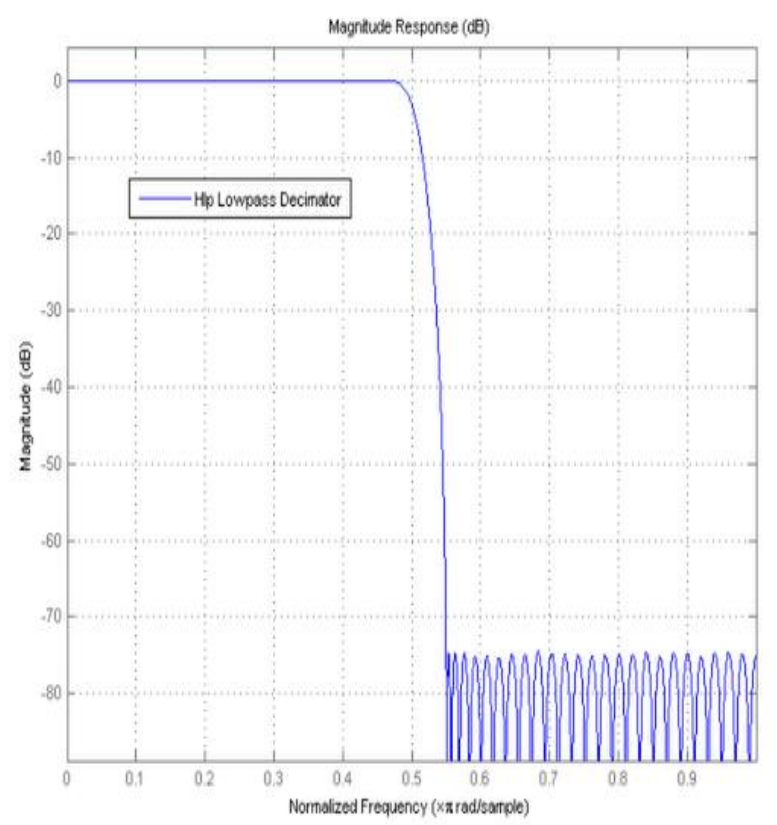

set(hfv, 'Filters', [Hlp,Hhp,Glp,Ghp]);

legend(hfv,'Hlp Lowpass Decimator','Hhp Highpass Decimator',...

'Glp Lowpass Interpolator','Ghp Highpass Interpolator');

For the sake of the demo let $\mathrm{p}[\mathrm{n}]$ denote the signal

$$
p[n]=\delta[n]+\delta[n-1]+\delta[n-2]
$$


and let the signal $x[\mathrm{n}]$ be defined by

$x[n]=p[n]+2 p[n-8]+3 p[n-16]+4 p[n-24]+3 p[n-32]+2 p[n-40]+p[n-48]$

NOTE: Since MATLAB ${ }^{\circledR}$ uses one-based indexing, delta[n]=1 when $\mathrm{n}=1$.

$\mathrm{x}=\operatorname{zeros}(512,1)$;

$\mathrm{x}(1: 3)=1 ; \mathrm{x}(8: 10)=2 ; \mathrm{x}(16: 18)=3 ; \mathrm{x}(24: 26)=4$;

$\mathrm{x}(32: 34)=3 ; \mathrm{x}(40: 42)=2 ; \mathrm{x}(48: 50)=1$;

stemplot(x,'x[n]');

set(gcf, 'Color', [ [ $\left.\left.\begin{array}{lll}1 & 1 & 1\end{array}\right]\right)$

The innovative aspects of the present work consist of estimating appropriate threshold by analyzing the statistical parameters of the wavelet coefficients. Our threshold is based on universal thresholding function. In this work, merits of interscale dependencies of adjacent scale wavelets are incorporated in the multiscale thresholding scheme for the purpose of denoising. In this scheme, two adjacent wavelet subbands are multiplied to amplify the significant features and condense noise. Then, thresholding is applied to the multiscale products instead of the wavelet

coefficients.

In general, a small threshold value will leave behind all the noisy coefficients, and subsequently the resultant denoised image may still be noisy. On the other hand, a large threshold value converts more number of coefficients to zero, which directs to smooth the signal, destroys details and the resultant image may cause blur and artifacts. Therefore, an optimum threshold value should be found out that is adaptive to different subband characteristics.

The original work of Donoho proposed universal threshold

$\lambda=\sigma_{n} \sqrt{\log 2 N}$

This threshold depends on the image size ( $\mathrm{N}$ ) and the noise standard deviation $\sigma_{\mathrm{n}}$. It is easy to implement over smooth images. Based on this, we proposed our threshold by estimating a parameter-weighted variance $(\delta)$. Instead of applying a preselected uniform threshold, we propose a background - support threshold selection scheme for a coefficient-dependent choice of the threshold. We define weighted variance for coefficient $\mathrm{Y}$ [ $\mathrm{m}, \mathrm{n}]$ for threshold determination. The parameter-weighted variances $\delta$ involve neighboring coefficients of the wavelet decomposition for the estimation of the local variance. It is based on the estimation of the local weighted variance $\sigma_{\mathrm{w}}[\mathrm{m}, \mathrm{n}$ ]$^{2}$ of each wavelet coefficient $\mathrm{Y}[\mathrm{m}, \mathrm{n}]$ at level 1 and orientation $\mathrm{O}$ using a window $\mathrm{N}$ of size $5 \times 5$. The weighted variance of a coefficient $\mathrm{Y}[\mathrm{m}, \mathrm{n}]$ with respect to the window of size $5 \times 5$ with weights $w=\left\{w_{i, j}, i, j \in N\right\}$ is defined by

$\delta=\sigma_{w}[m, n]^{2}=\frac{\sum_{i, j \in N} w_{i, j} Y[m, n]^{2}}{\sum_{i, j \in N} w_{i, j}}$

$\lambda(m, n)=\frac{\sigma_{n}^{2}}{\delta}$

After finding the threshold, the coefficients are thresholded by

$$
\begin{aligned}
& \hat{Y}_{L} f(m, n)=Y_{L} f(m, n) \text { if } P_{L} f(m, n) \geq t_{L} \hat{Y}_{L} f(m, n)=0 \\
& \text { if } P_{L} f(m, n)<t_{L}
\end{aligned}
$$

if

$L \quad=\quad 1$

$\underline{2}$,

$K$

Multiscale product is obtained by multiplying the adjacent scale wavelet coefficients. In our work, we perform two levels of decomposition, and the product is taken between adjacent scale wavelet coefficients. For 2-D images, the multiscale products have two components

$$
\begin{aligned}
& P_{L}^{m} f(m, n)=w_{L}^{m} f(m, n)^{\star} w_{L+1}^{m} f(m, n) \\
& P_{L}^{n} f(m, n)=w_{L}^{n} f(m, n)^{*} w_{L+1}^{n} f(m, n)
\end{aligned}
$$

The parameter noise variance $\sigma^{2}$ needs to be estimated first. It may be possible to measure $\sigma^{2}$ based on information other than the corrupted image, and it is estimated from the subband $\mathrm{HH}_{1}$ by the robust median estimator,

$$
\left.\sigma^{2}=\left[\frac{\text { median } \mid}{0.6745}\right]_{m, n} \mid\right]^{2}
$$

Weighted (9) variance (d) of a given wavelet coefficient is determined by the weight in a local window.

\section{Challenges and Discussion}

In order to study the performance of matched wavelets w.r.t the frequency content of the image, the test images were also classified by their frequency distribution. For doing so, the PSD of the given image is calculated in three intervals of full spectrum such as i) 0 to $\_/ 3$, ii) $\_/ 3$ to $2 \_/ 3$ and iii) $2 \_/ 3$ to _. Now the images were classified as low pass, band pass and high pass when PSD is maximum in interval1, interval2 and interval 3 respectively. The experiments were carried on the set of low pass and high pass images only, because they are the ones which are found more often in general and in our datasets. We have also made comparisons with the Wiener filter, the best linear filtering possible. The version used is the adaptive filter, wiener2, in the MATLAB image processing toolbox, using the default settings The PSNR results are shown in Table 1, and they are considerably worse than the nonlinear thresholding methods, especially when $\sigma$ is large. The image quality is also not as good as those of the thresholding methods. It is clear from Table 1, that the proposed thresholding technique outperforms the VisuShrink and the filters like wiener. The proposed method removes noise significantly and remains within $4 \%$ of the oracle shrink.

\section{GONCLUSION DIRECTION}

AND

FUTURE

In this paper, a simple and subband adaptive threshold is proposed to address the issue of image recovery from its noisy counterpart. It is based on the generalized Guassian distribution modeling of subband coefficients. The image denoising 
algorithm uses soft thresholding to provide smoothness and better edge preservation at the same time. We also explored the utility of using image-matched partial biorthogonal filters for denoising. Our design of image-matched biorthogonal wavelet bases uses the constraint that most of the energy of clean image is projected into scaling subspace rather than the wavelet subspace. We have compared denoising performance of our matched wavelets with CDF biorthogonal wavelets with two well-known thresholding strategies for various image datasets. The results show that adapted biorthogonal wavelets performed much better denoising than the available biorthogonal wavelets for low SNR i.e where the actual need for adaptation arises.

\section{REFERENCES}

[1] D. L. Donoho and I. M. Johnstone. Ideal spatial adaptation by wavelet shrinkage. Biometrika, 81:425-455, March 1994.

[2] G. Chang, B. Yu, and M. Vetterli. Adaptive wavelet thresholding

for image denoising and compression. IEEE Transa-tions on Image Processing, 9:1532-1546, September 2000.

[3] L. Sendur and I.W. Selesnick. Bivariate shrinkage functions for wavelet-based denoising exploiting interscale dependency.IEEE Transactions on Signal Processing, 50:27442756, November 2002

[4] J. Portilla, V. Strela, M. Wainwright, and E. Simoncelli. Image denoising using scale mixtures of gaussians in the wavelet domain. IEEE Transactions on Image Processing,12:1338-1351, November 2003
[5] J. O. Chapa and R. M. Rao. Algorithms for designing wavelets to match a specified signal. IEEE Transactions on Signal Processing, 2000

[6] A. H. Twefik, D. Sinha, and P. Jorgensen. On the optimal choice of a wavelet for signal representation. IEEE Transactions on Information Theory, 38:747-765, February 1992.

[7] A. Gupta, S. D. Joshi, and S. Prasad. A new approach for estimation of statistically matched wavelet. IEEE Transactions on Signal Processing, 53:1778-1793, May 2005.

[8] I. Daubechies. (1992). “Ten Lectures on Wavelets", Philadelphia SIAM.

[9] M. S. Crouse, R. D. Nowak, and R. G. Baraniuk, "Waveletbased statistical signal processing-Markov models,"IEEE Trans. Signal Processing, vol. 46, no. 4, pp. 886-902, April 1998.

[10] J. K. Romberg, H. Choi, and R. Baraniuk, "Bayesian treestructured image modeling using wavelet-domain hidden Markov model," in Proc. of SPIE, Denver, CO, July 1999, vol. 3816, pp. 31 .

[11] S. Mallat. A theory for multiresolution signal decomposition:The wavelet representation. IEEE Trans. Pattern Anal.Mach. Intell., 11:674-693, July 1989.

[12] A. Gupta, S. D. Joshi, and S. Prasad. A new approach for estimation of statistically matched wavelet. IEEE Transactions on Signal Processing, 53:1778-1793, May 2005. 